\title{
A New Tandem Reaction of Benzyne: One-Pot Synthesis of Aryl Amines Containing Anthracene
}

\author{
Chunsong Xie, Yuhong Zhang.* \\ Department of Chemistry, Zhejiang University, Hangzhou 310027, P.R. China. \\ E-mail: yhzhang@zjuem.zju.edu.cn
}

Supporting Information

\section{Content}

General procedure of the tandem reaction......................S2

Characterization data of the product............................S3

GC-MS analysis of the reaction solution of o-trimethylsilyl aryltriflate

with 1-ethyl-4-phenyl-1 $H$-imidazole $(\mathrm{T} 2-12) \ldots \ldots \ldots \ldots \ldots \ldots \ldots \ldots . . . \ldots \ldots \ldots$

References............................................... 22 
General All the reactions were carried out under the nitrogen atmosphere in oven-dried flask. $\mathrm{CH}_{3} \mathrm{CN}$ was distilled from $\mathrm{CaH}_{2}$ using benzophenone as the indicator. O-(trimethylsilyl)phenyl triflate was prepared according to the method of reference 1. N-ethylimidazole, N-butylimidazole, N-allylimidazole, N-benzylimidazole, 1,2-dimethylimidazole, 1-ethyl-2-phenyimidazole and 1-ethyl-4-phenylimidazole were prepared according to the method of reference 2. N-phenylimidazole, 1-(4-methoxyphenyl)imidazole, 1-tolylimidazole, 1-(4-chlorophenyl)imidazole, 1-(4-(trifloromethyl)phenyl)imidazole and 1,2-diphenylimidazole was prepared according to the method of reference 3 . The other materials were purchased from common commercial sources and used without additional purification. ${ }^{1} \mathrm{H}$ NMR spectra were recorded at $400 \mathrm{MHz}$ using TMS as internal standard. ${ }^{13} \mathrm{C}$ NMR spectra were recorded at $100 \mathrm{MHz}$ using TMS as internal standard. Mass spectroscopy data of the product of the tandem reaction was collected on an HRMS-APCI instrument. Infrared spectra were obtained from a FTIR spectrometer.

Representative procedure of the tandem reaction: $o$-(Trimethylsilyl)phenyl triflate $(149 \mathrm{mg}$, $0.5 \mathrm{mmol})$ in $\mathrm{CH}_{3} \mathrm{CN}(2 \mathrm{~mL})$ was added dropwise to a suspension of $\mathrm{CsF}(152 \mathrm{mg}, 1.0 \mathrm{mmol})$ and $\mathrm{N}$-substituted imidazole $(41 \mathrm{mg}, 0.5 \mathrm{mmol})$ in $3 \mathrm{~mL} \mathrm{CH}{ }_{3} \mathrm{CN}$. The reaction mixture was allowed to reaction at $50{ }^{\circ} \mathrm{C}$ for $12 \mathrm{~h}$. After the reaction, the reaction mixture was cooled to room temperature and filtered through a pad of cellite. The filtrate was distilled under reduced pressure, and the product was obtained by flash chromatography on a silica gel column. 
(1) N-methyl-N-phenylanthracen-10-amine [ T 1-2, 112222-72-7 ]

${ }^{1} \mathrm{H}$ NMR ( $400 \mathrm{MHz}, \mathrm{CDCl}_{3}$, TMS ) $\delta 8.47(\mathrm{~s}, 1 \mathrm{H}$ ), 8.07 ( d, $J=8 \mathrm{~Hz}, 2 \mathrm{H}$ ), $7.90(\mathrm{~d}, J=12.4$ $\mathrm{Hz}, 2 \mathrm{H}$ ), 7.47 ( t, $J=7.4 \mathrm{~Hz}, 2 \mathrm{H}$ ), 7.41 ( t, $J=7.6 \mathrm{~Hz}, 2 \mathrm{H}$ ), 7.13 ( m, $2 \mathrm{H}$ ), 6.88 ( t,$J=7.4$ $\mathrm{Hz}, 1 \mathrm{H}$ ), 6.48 ( m, $2 \mathrm{H}$ ), 3.53 ( s, $3 \mathrm{H}$ ); ${ }^{13} \mathrm{C} \mathrm{NMR}\left(100 \mathrm{MHz}, \mathrm{CDCl}_{3}\right.$ ) $\delta 149.9,139.3,134.1$, $132.8,129.8,129.2,128.9,126.3,126.2,125.5,124.1,116.5,39.3 . \mathrm{IR}(\mathrm{KBr}) v=3051,2925$, 2801, 1599, 1499, 1482, 1373, 1107, 845, 743, $687 \mathrm{~cm}^{-1}$; HRMS (APCI) Calcd for $\mathrm{C}_{21} \mathrm{H}_{17} \mathrm{~N}$ : $[\mathrm{M}+\mathrm{H}]^{+}$284.1434; Found, 284.1419.
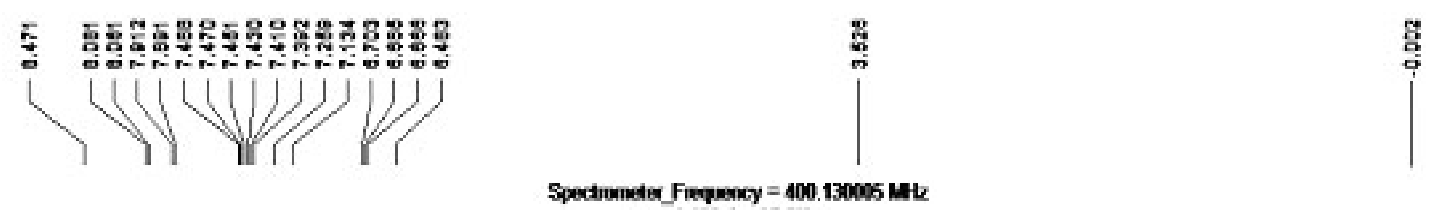
xist-1-199 in CDCB

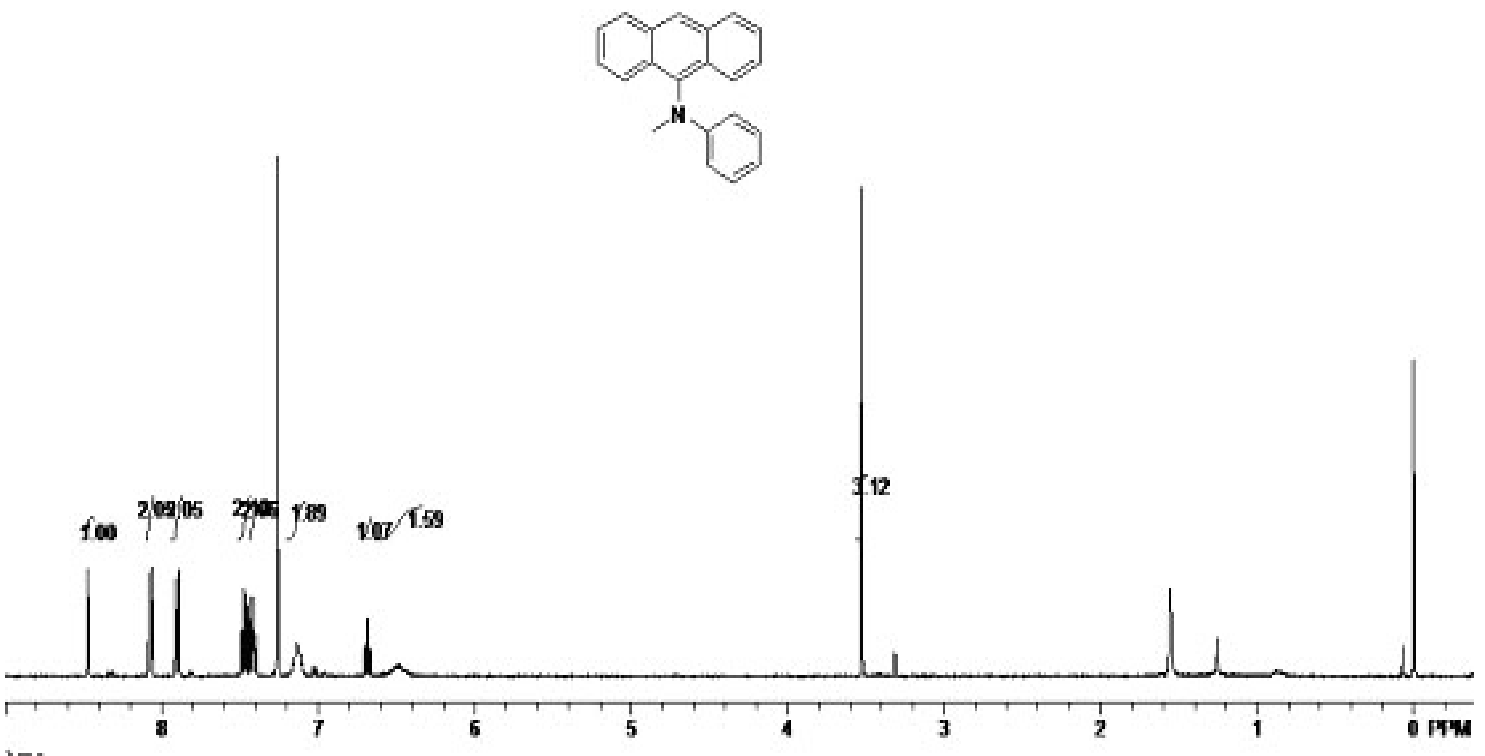




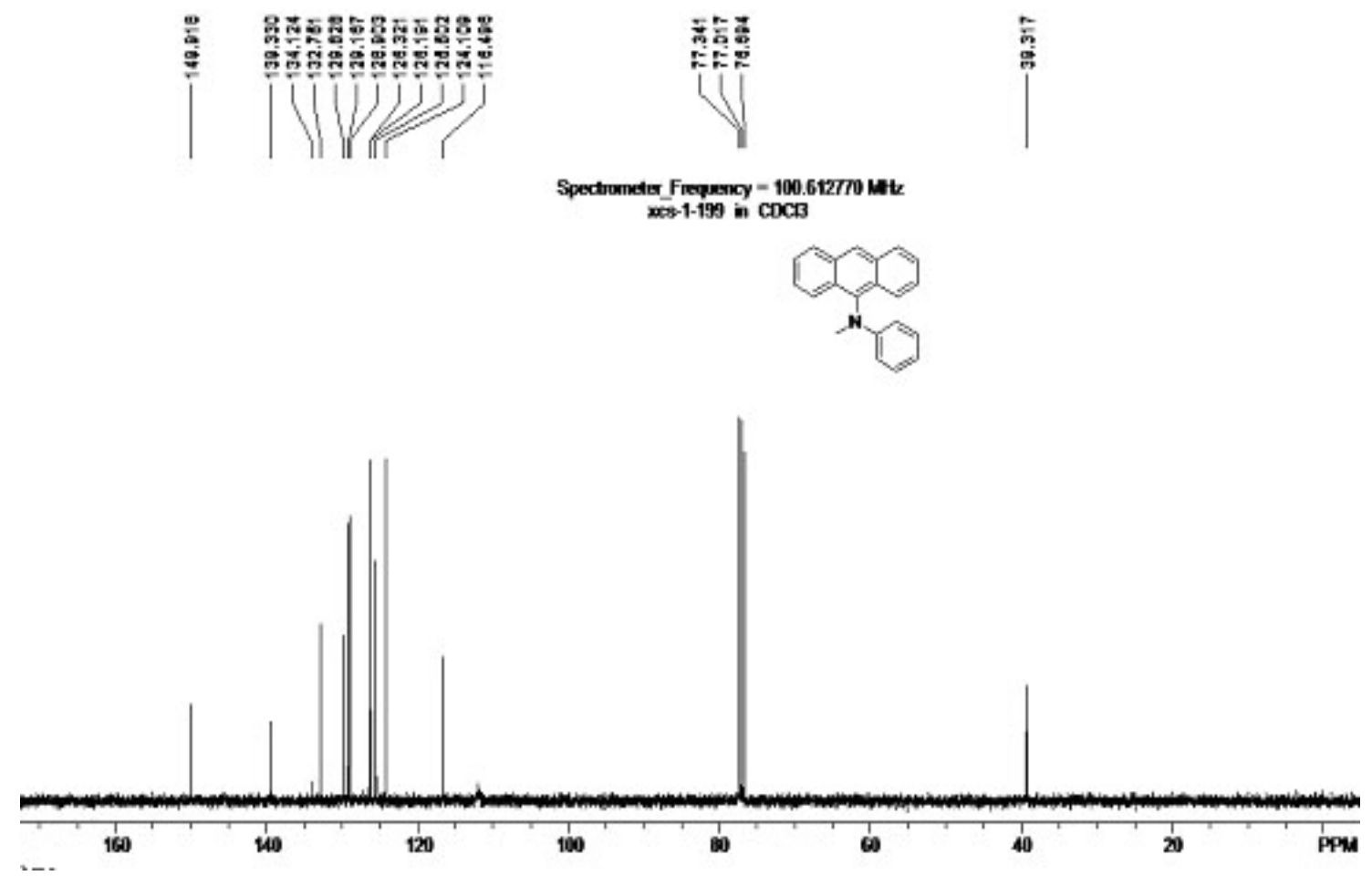

(2) N-ethyl-N-phenylanthracen-10-amine [ T 2-1, New compound ]

${ }^{1} \mathrm{H}$ NMR ( $400 \mathrm{MHz}, \mathrm{CDCl}_{3}$, TMS ) $\delta 8.48(\mathrm{~s}, 1 \mathrm{H}$ ), 8.07 ( d, J=8.4 Hz, $2 \mathrm{H}$ ), 7.96 ( d, $J=8.8$ $\mathrm{Hz}, 2 \mathrm{H}$ ), 7.47 ( t, J=7.2 Hz, $2 \mathrm{H}$ ), 7.41 ( t, $J=8.2 \mathrm{~Hz}, 2 \mathrm{H}$ ), 7.12 ( t, $J=7.8 \mathrm{~Hz}, 2 \mathrm{H}$ ), 6.67 ( t, $J=7.4 \mathrm{~Hz}, 1 \mathrm{H}), 6.49(\mathrm{~d}, J=7.6 \mathrm{~Hz}, 2 \mathrm{H}), 3.98(\mathrm{q}, 2 \mathrm{H}), 1.28(\mathrm{t}, J=7,3 \mathrm{H}) .{ }^{13} \mathrm{C} \mathrm{NMR}(100$ $\left.\mathrm{MHz}, \mathrm{CDCl}_{3}\right) \delta 148.9,137.7,132.3,130.0,128.8,128.4,126.8,125.8,125.0,124.1,120.5$, 115.9, 46.8, 13.1. IR ( KBr ) v = 3049, 2925, 2853, 1597, 1499, 1441, 1412, 1275, 889, 847, 791, 742, 692. HRMS (APCI) Calcd for $\mathrm{C}_{22} \mathrm{H}_{19} \mathrm{~N}$ : $[\mathrm{M}+\mathrm{H}]^{+}$298.1590; Found, 298.1576. 

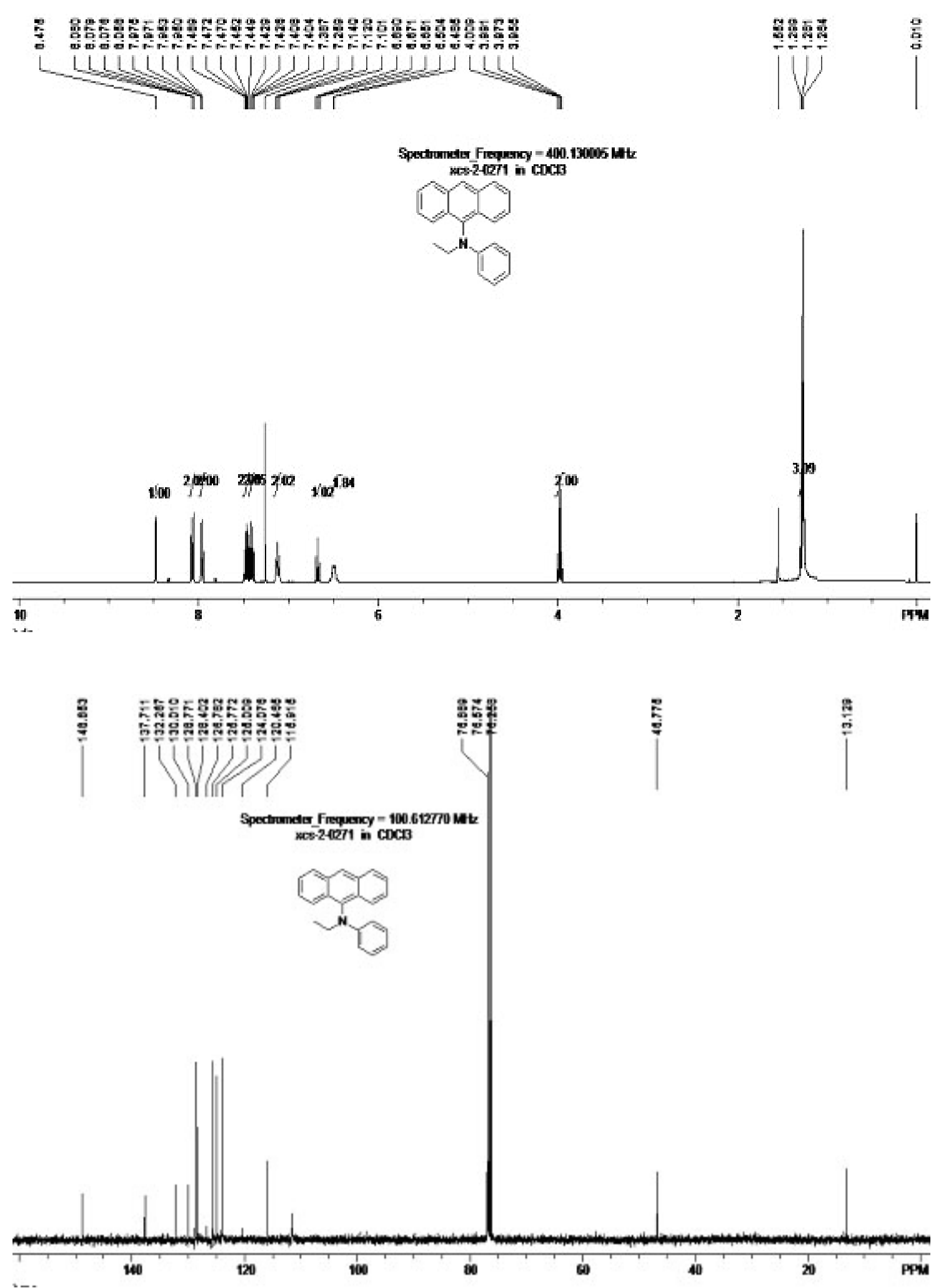
(3) N-butyl-N-phenylanthracen-10-amine [ T 2-2, New compound ]

${ }^{1} \mathrm{H}$ NMR ( $400 \mathrm{MHz}, \mathrm{CDCl}_{3}$, TMS ) $\delta 8.48(\mathrm{~s}, 1 \mathrm{H}$ ), 8.07 ( d, $J=8 \mathrm{~Hz}, 2 \mathrm{H}$ ), 7.95 ( d, $J=8.4$ $\mathrm{Hz}, 2 \mathrm{H}$ ), 7.47 ( t, $J=7.4 \mathrm{~Hz}, 2 \mathrm{H}$ ), 7.41 ( t $, J=7.6 \mathrm{~Hz}, 2 \mathrm{H}$ ), 7.12 ( t $, J=8 \mathrm{~Hz}, 2 \mathrm{H}$ ), 6.67 ( t, $J=7.4 \mathrm{~Hz}, 1 \mathrm{H}$ ), $6.46(\mathrm{~d}, J=3.8 \mathrm{~Hz}, 2 \mathrm{H}), 3.86(\mathrm{~m}, 2 \mathrm{H}), 1.78(\mathrm{~m}, 2 \mathrm{H}), 1.29(\mathrm{~m}, 2 \mathrm{H}), 0.88$ $(\mathrm{t}, J=6.4,3 \mathrm{H}) .{ }^{13} \mathrm{C}$ NMR $\left(100 \mathrm{MHz}, \mathrm{CDCl}_{3}\right) \delta 149.3,138.5,132.7,130.2,129.1,128.8$, 127.2, 126.1, 125.4, 124.4, 118.0, 116.3, 53.2, 30.2, 20.3, 13.8. IR ( KBr ) v = 2955, 2928, 2869, 1597, 1500, 1412, 1374, 891, 845, 741, 691. HRMS (APCI) Calcd for $\mathrm{C}_{24} \mathrm{H}_{23} \mathrm{~N}:[\mathrm{M}+\mathrm{H}]^{+}$ 326.1903; Found, 326.1887.
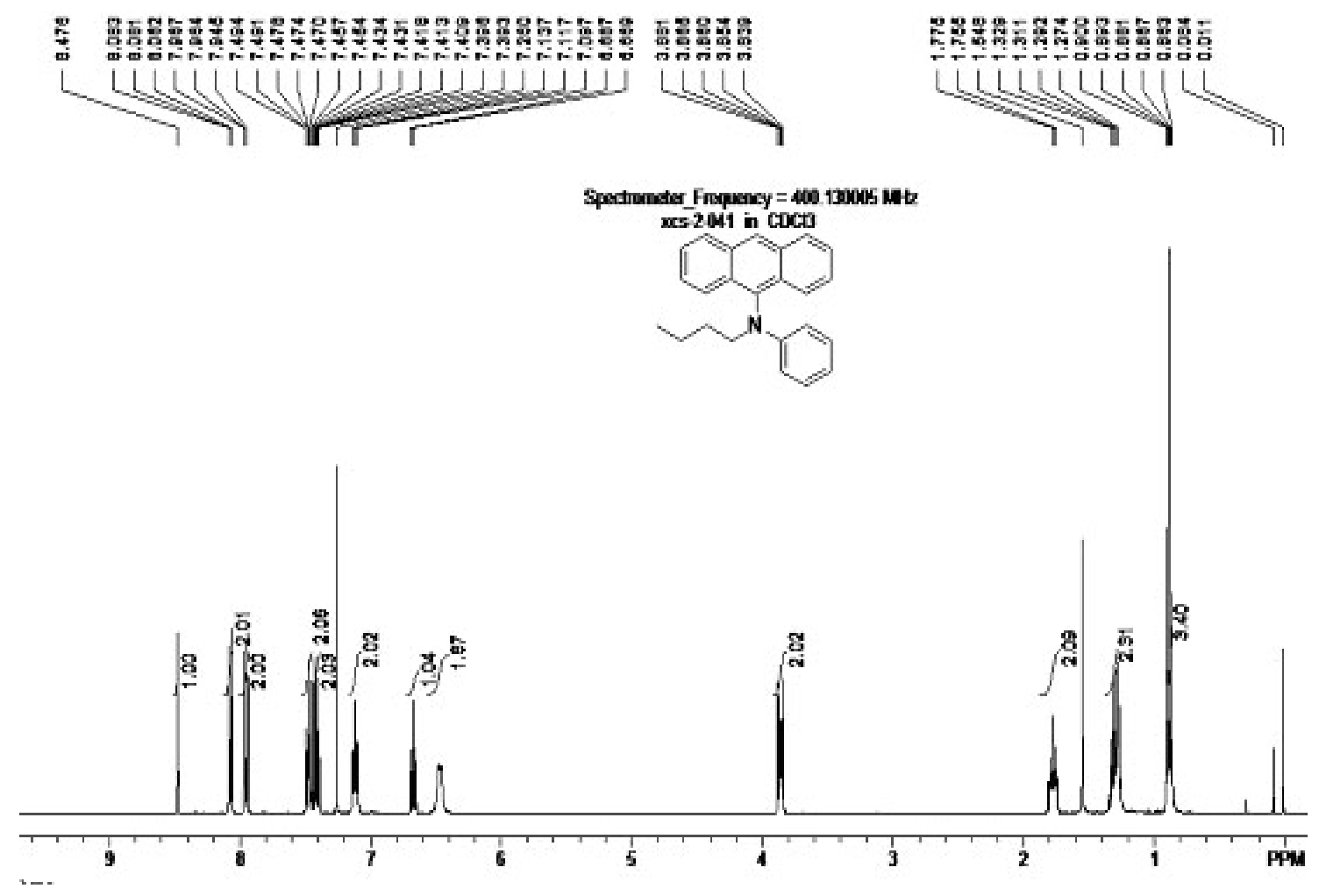


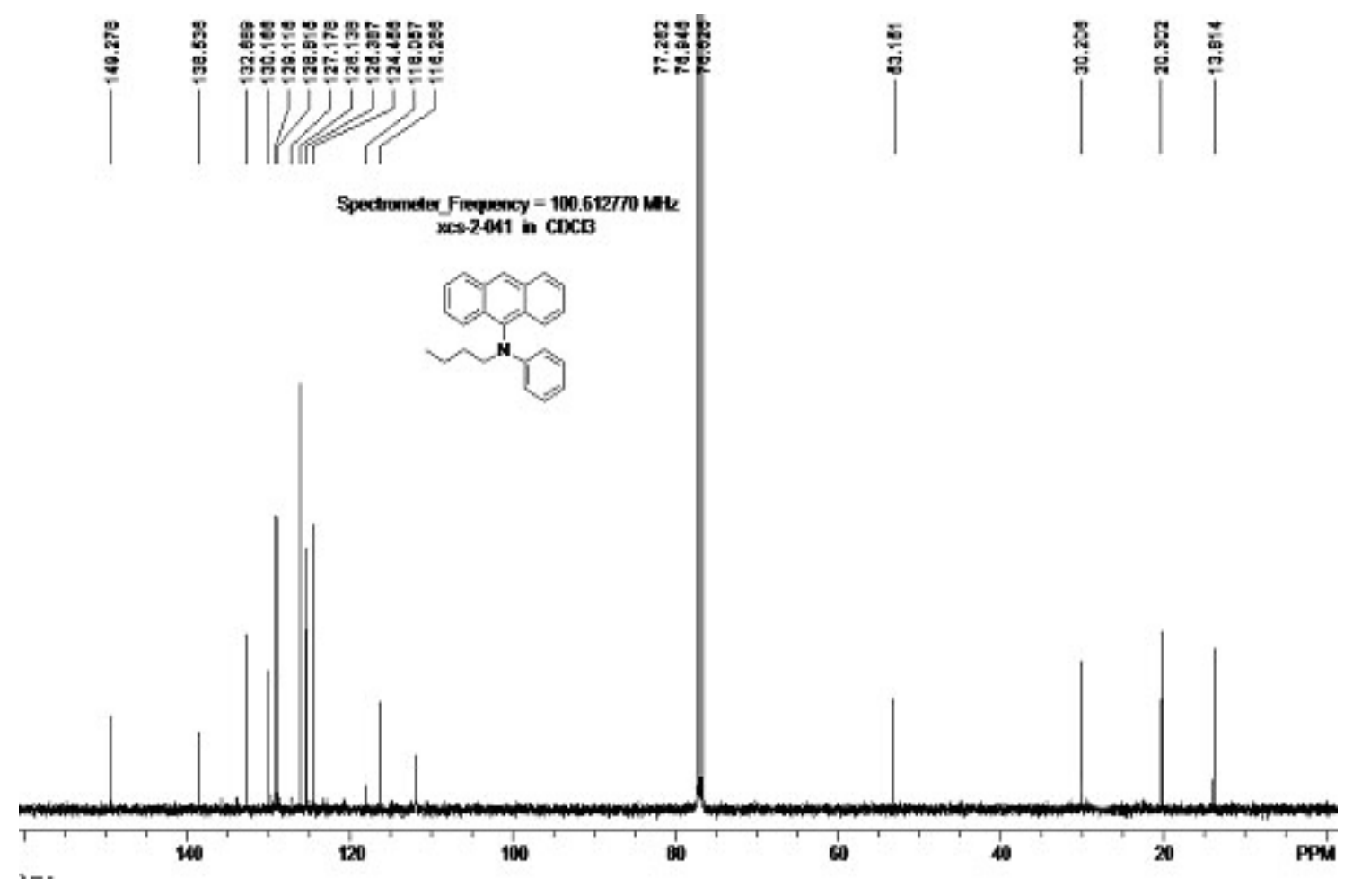

(4) N-allyl-N-phenylanthracen-10-amine [ T 2-3, New compound ]

${ }^{1} \mathrm{H}$ NMR ( $400 \mathrm{MHz}, \mathrm{CDCl}_{3}$, TMS ) $\delta 8.48(\mathrm{~s}, 1 \mathrm{H}$ ), 8.07 ( d, $J=8 \mathrm{~Hz}, 2 \mathrm{H}$ ), 7.98 ( d, $J=8.8$ $\mathrm{Hz}, 2 \mathrm{H}$ ), $7.47(\mathrm{t}, J=7.2 \mathrm{~Hz}, 2 \mathrm{H}$ ), $7.41(\mathrm{t}, J=7.4 \mathrm{~Hz}, 2 \mathrm{H}$ ), 7.12 ( t, $J=8 \mathrm{~Hz}, 2 \mathrm{H}$ ), 6.70 ( t, $J=7.4 \mathrm{~Hz}, 1 \mathrm{H}$ ), 6.53 ( d, $J=6.4 \mathrm{~Hz}, 2 \mathrm{H}$ ), $6.13(\mathrm{~m}, 1 \mathrm{H}), 5.23$ ( d, $J=17.6 \mathrm{~Hz}, 1 \mathrm{H}$ ), 5.12 ( d, $J=10.4 \mathrm{~Hz}, 1 \mathrm{H}), 4.50(\mathrm{~d}, J=6 \mathrm{~Hz}, 2 \mathrm{H}) .{ }^{13} \mathrm{C} \mathrm{NMR}\left(100 \mathrm{MHz}, \mathrm{CDCl}_{3}\right) \delta 149.5,138.5,134.8$, $132.7,130.1,129.1,128.8,126.4,126.2,125.4,124.4,117.4,116.8,112.6,56.0$. IR ( KBr ) $v=$ 2951, 2926, 2854, 1598, 1500, 1407, 1370, 1249, 887, 741, 683. HRMS (APCI) Calcd for $\mathrm{C}_{23} \mathrm{H}_{19} \mathrm{~N}:[\mathrm{M}+\mathrm{H}]^{+}$310.1590; Found, 310.1574 . 


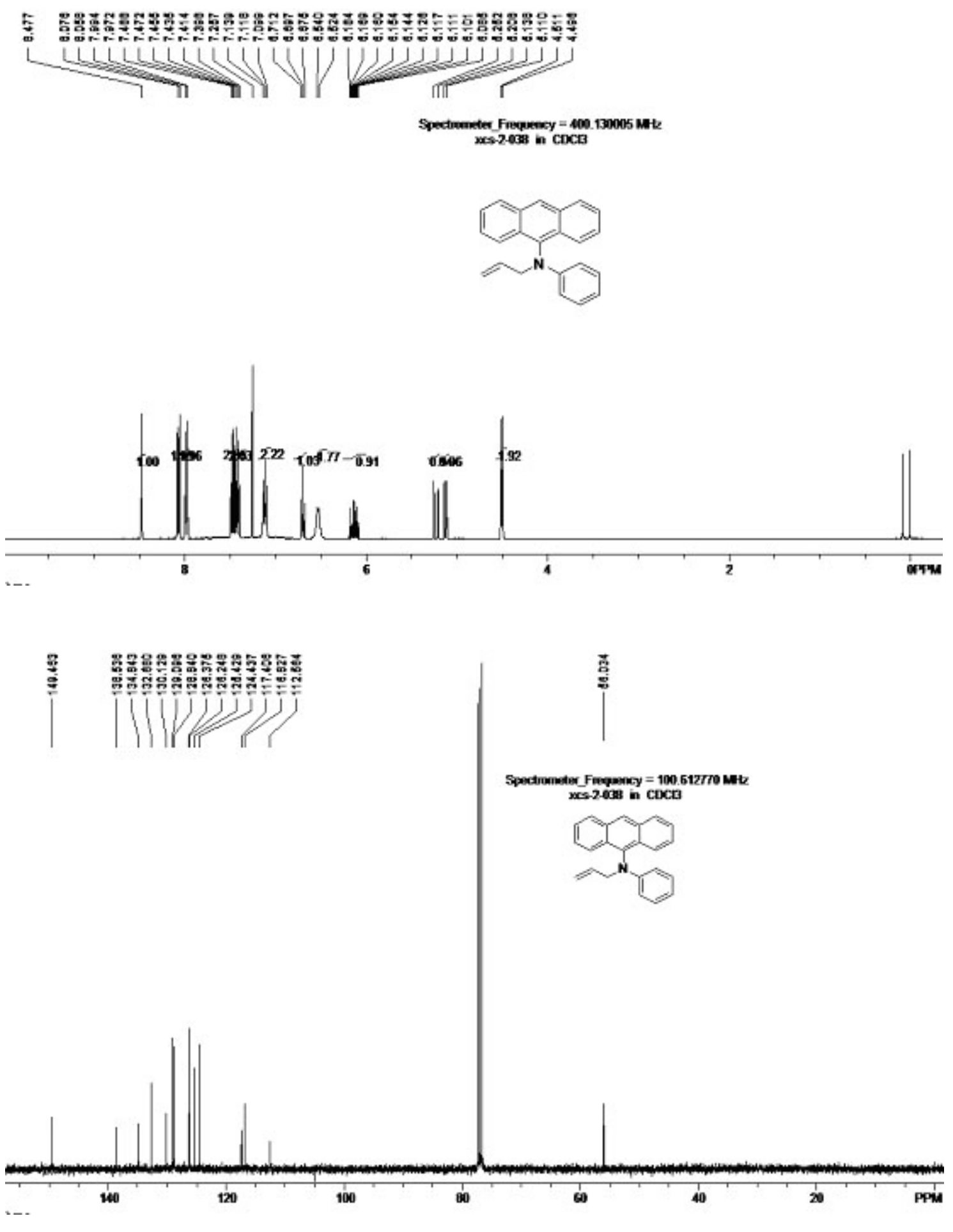




\section{(5) N-benzyl-N-phenylanthracen-10-amine [ T 2-4, New compound ]}

${ }^{1} \mathrm{H}$ NMR ( $400 \mathrm{MHz}, \mathrm{CDCl}_{3}$, TMS ) $\delta 8.46(\mathrm{~s}, 1 \mathrm{H}$ ), 8.05 ( d, $J=8.4 \mathrm{~Hz}, 2 \mathrm{H}$ ), 7.97 ( d, $J=8.4$ $\mathrm{Hz}, 2 \mathrm{H}$ ), 7.45 ( t, $J=7.4 \mathrm{~Hz}, 2 \mathrm{H}$ ), 7.37 ( t, $J=7.6 \mathrm{~Hz}, 2 \mathrm{H}$ ), 7.33 ( t, $J=6.6 \mathrm{~Hz}, 2 \mathrm{H}$ ), 7.20 ( m, $3 \mathrm{H}$ ), 7.10 ( t, $J=8.2 \mathrm{~Hz}, 2 \mathrm{H}$ ), 6.70 ( t, $J=7 \mathrm{~Hz}, 1 \mathrm{H}$ ), 6.61 ( d, $J=8 \mathrm{~Hz}, 2 \mathrm{H}$ ), 5.10 ( s, $1 \mathrm{H}$ ). ${ }^{13} \mathrm{C}$ NMR ( $100 \mathrm{MHz}, \mathrm{CDCl}_{3}$ ) $\delta 149.6,138.8,138.4,133.7,132.3,129.6,128.7,127.8,125.7$, 124.9, 124.2, 116.8, 112.6, 57.2. IR ( KBr ) $v=3059$, 3030, 2930, 1597, 1497, 1407, 1377, 1367, 1249, 751, 741, 734, 691. HRMS (APCI) Calcd for $\mathrm{C}_{27} \mathrm{H}_{21} \mathrm{~N}$ : $[\mathrm{M}+\mathrm{H}]^{+}$360.1747; Found, 360.1729 .

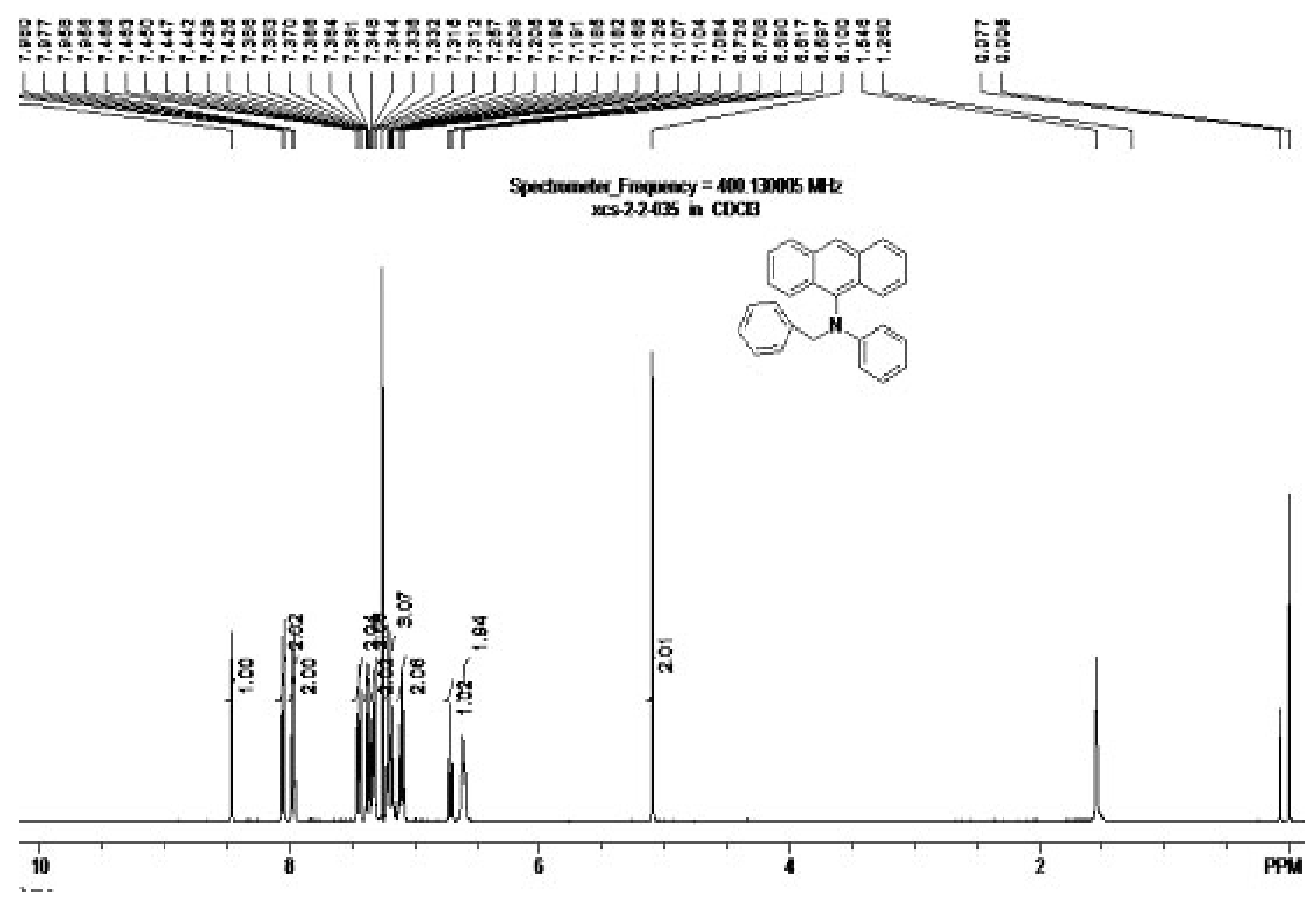




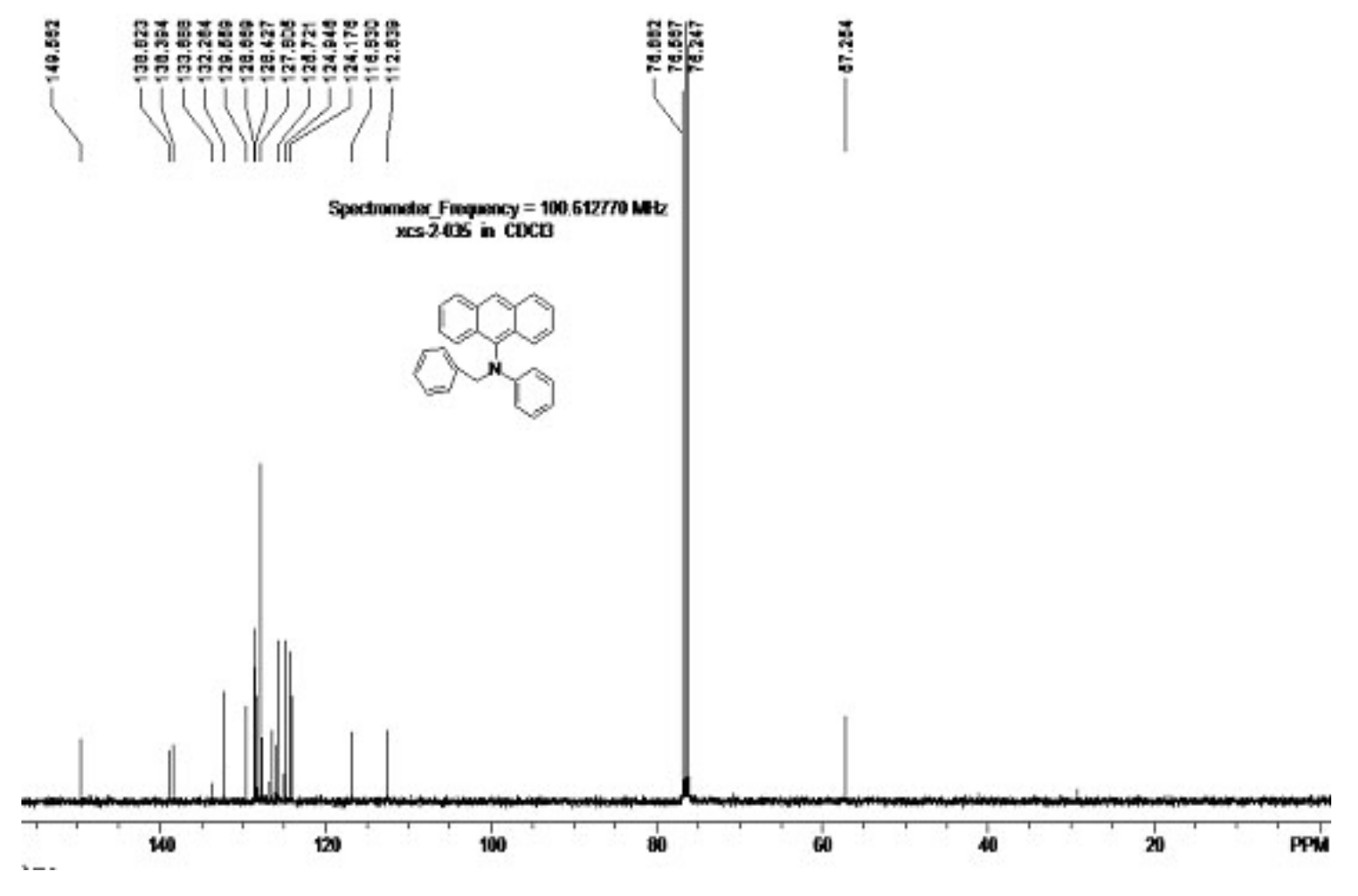

(6) N,N-diphenylanthracen-10-amine [ T 2-5, 62770-62-1 Ref. 4 ]

${ }^{1} \mathrm{H}$ NMR ( $400 \mathrm{MHz}, \mathrm{CDCl}_{3}$, TMS ) $\delta 8.51(\mathrm{~s}, 1 \mathrm{H}$ ), 8.13 ( d, $J=8.4 \mathrm{~Hz}, 2 \mathrm{H}$ ), 8.06 ( d, $J=8.4$ $\mathrm{Hz}, 2 \mathrm{H}$ ), 7.45 ( t, J=7.6 Hz, $2 \mathrm{H}$ ), 7.38 ( t $, J=7.8 \mathrm{~Hz}, 2 \mathrm{H}$ ), 7.15 ( t, J=7.8 Hz, $4 \mathrm{H}$ ), 7.07 ( d, $J=8.8 \mathrm{~Hz}, 4 \mathrm{H}$ ), 6.87 ( $\mathrm{t}, J=7.4 \mathrm{~Hz}, 2 \mathrm{H}) .{ }^{13} \mathrm{C} \mathrm{NMR}\left(100 \mathrm{MHz}, \mathrm{CDCl}_{3}\right) \delta 147.1,136.5,132.2$, 130.0, 128.5, 128.2, 126.1, 124.8, 123.7, 120.5, 111.6. IR ( KBr ) v = 3055, 2924, 2847, 1586, 1494, 1361, 1291, 891, 787, 751, 737, 683. HRMS (APCI) Calcd for $\mathrm{C}_{26} \mathrm{H}_{19} \mathrm{~N}:[\mathrm{M}+\mathrm{H}]^{+}$ 345.1513; Found, 345.1517. 

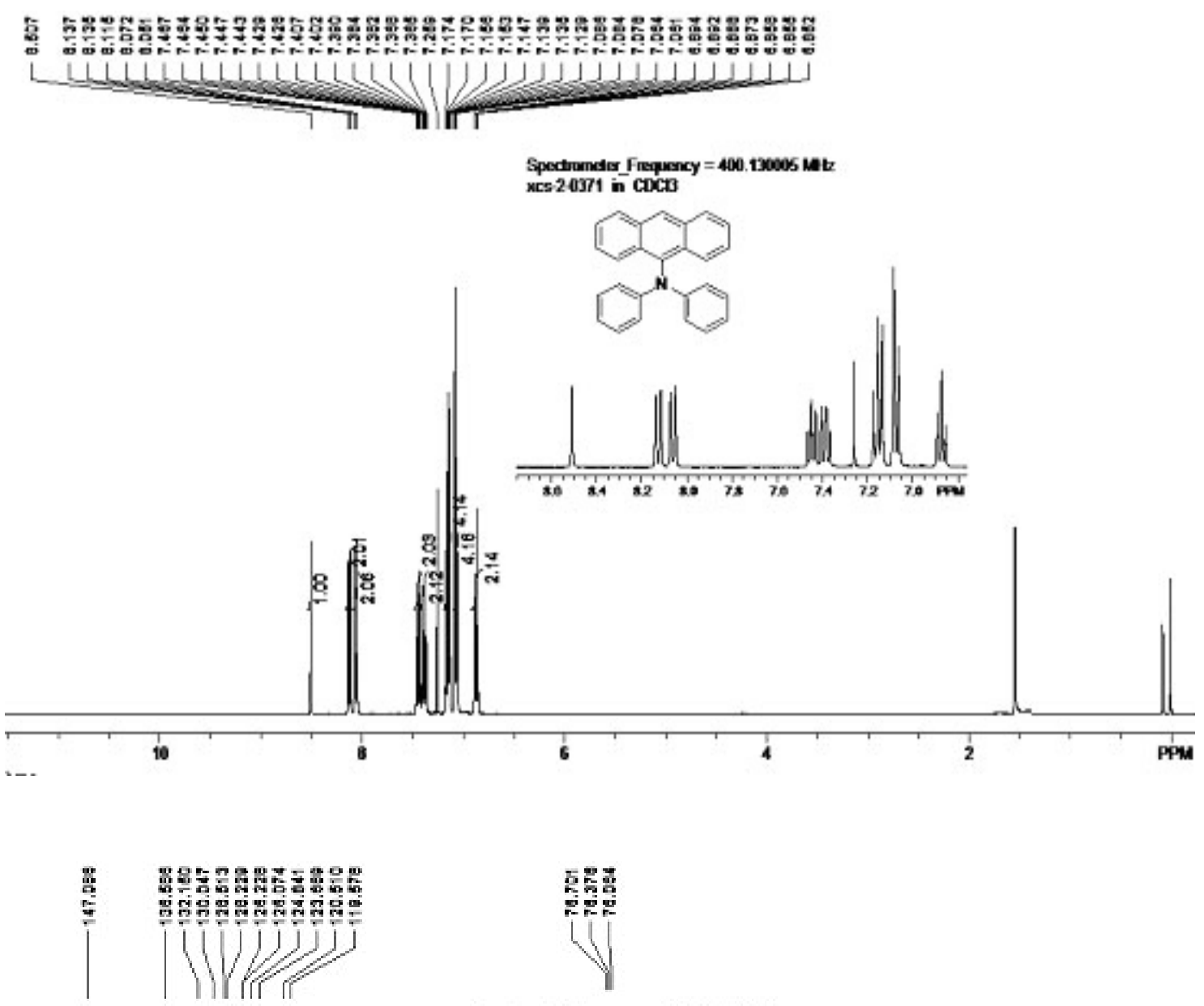

Spectreneder Frepuency $=100.612 T / 0$ Mht xus-2-0371 is CDCB

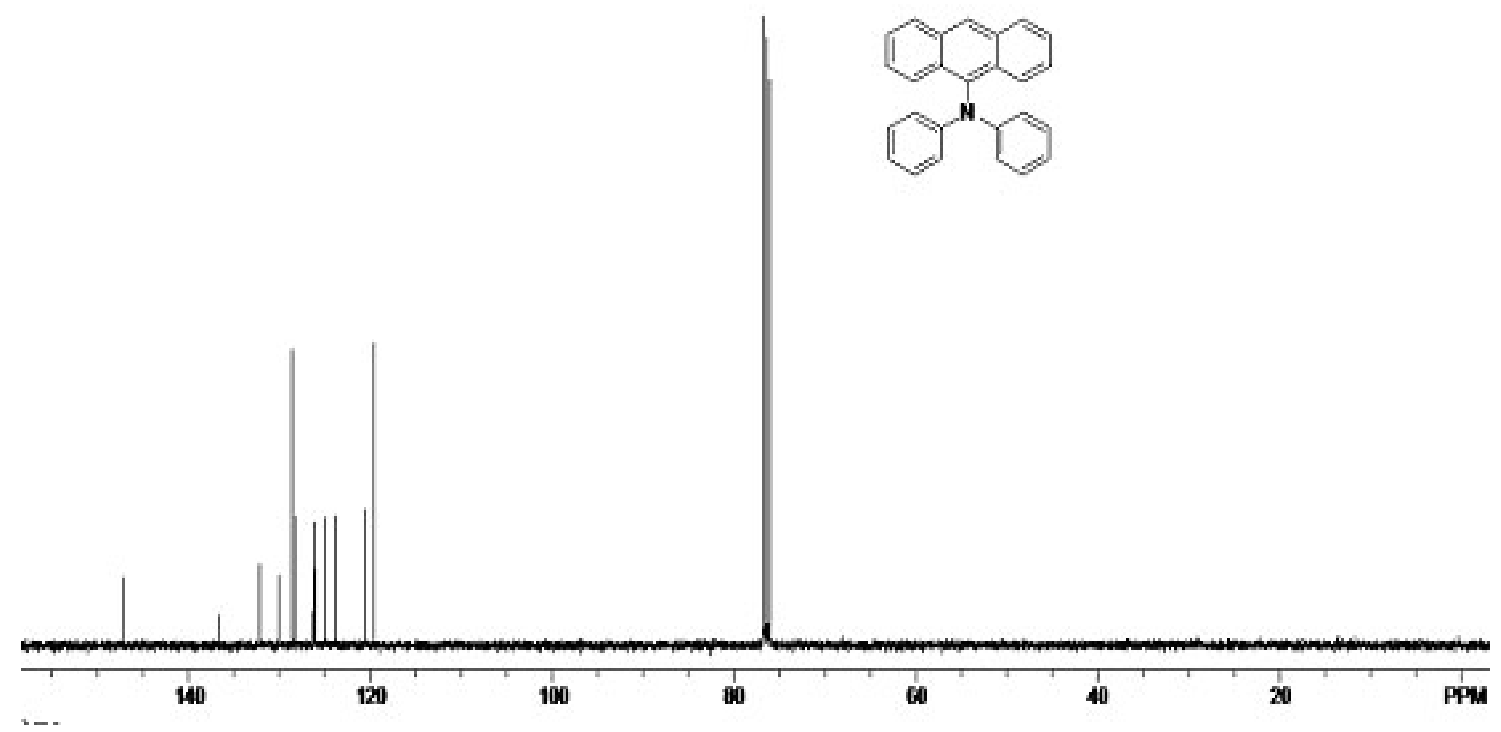


(7) N-(4-methoxyphenyl)-N-phenylanthracen-10-amine [ T 2-6, New compound ]

${ }^{1} \mathrm{H}$ NMR ( $400 \mathrm{MHz}, \mathrm{CDCl}_{3}$, TMS ) $\delta 8.48(\mathrm{~s}, 1 \mathrm{H}$ ), $8.13(\mathrm{~d}, J=8.4 \mathrm{~Hz}, 2 \mathrm{H}$ ), 8.04 ( d, $J=8$ $\mathrm{Hz}, 2 \mathrm{H}), 7.43(\mathrm{t}, J=7.4 \mathrm{~Hz}, 2 \mathrm{H}), 7.38(\mathrm{t}, J=7.6 \mathrm{~Hz}, 2 \mathrm{H}), 7.11(\mathrm{~m}, 4 \mathrm{H}), 6.91(\mathrm{~d}, J=8.8$ $\mathrm{Hz}, 2 \mathrm{H}$ ), 6.79 ( t $J=7.4 \mathrm{~Hz}, 1 \mathrm{H}$ ), 6.73 ( d, $J=8.8 \mathrm{~Hz}, 2 \mathrm{H}$ ), $3.71(\mathrm{~s}, 3 \mathrm{H}) .{ }^{13} \mathrm{C} \mathrm{NMR}(100$ $\left.\mathrm{MHz}, \mathrm{CDCl}_{3}\right) \delta 154.7,148.7,140.7,137.5,132.8,130.6,129.1,128.8,126.5,125.4,124.4$, 123.0, 122.2, 119.8, 118.3, 114.5, 55.4. IR ( KBr ) $v=3052,2955,2923,2852,1593,1508$, 1493, 1358, 1248, 1030, 837, 782, 746, 691, 608. HRMS (APCI) Calcd for $\mathrm{C}_{27} \mathrm{H}_{21} \mathrm{NO}:[\mathrm{M}+\mathrm{H}]^{+}$ 376.1696; Found, 376.1678.

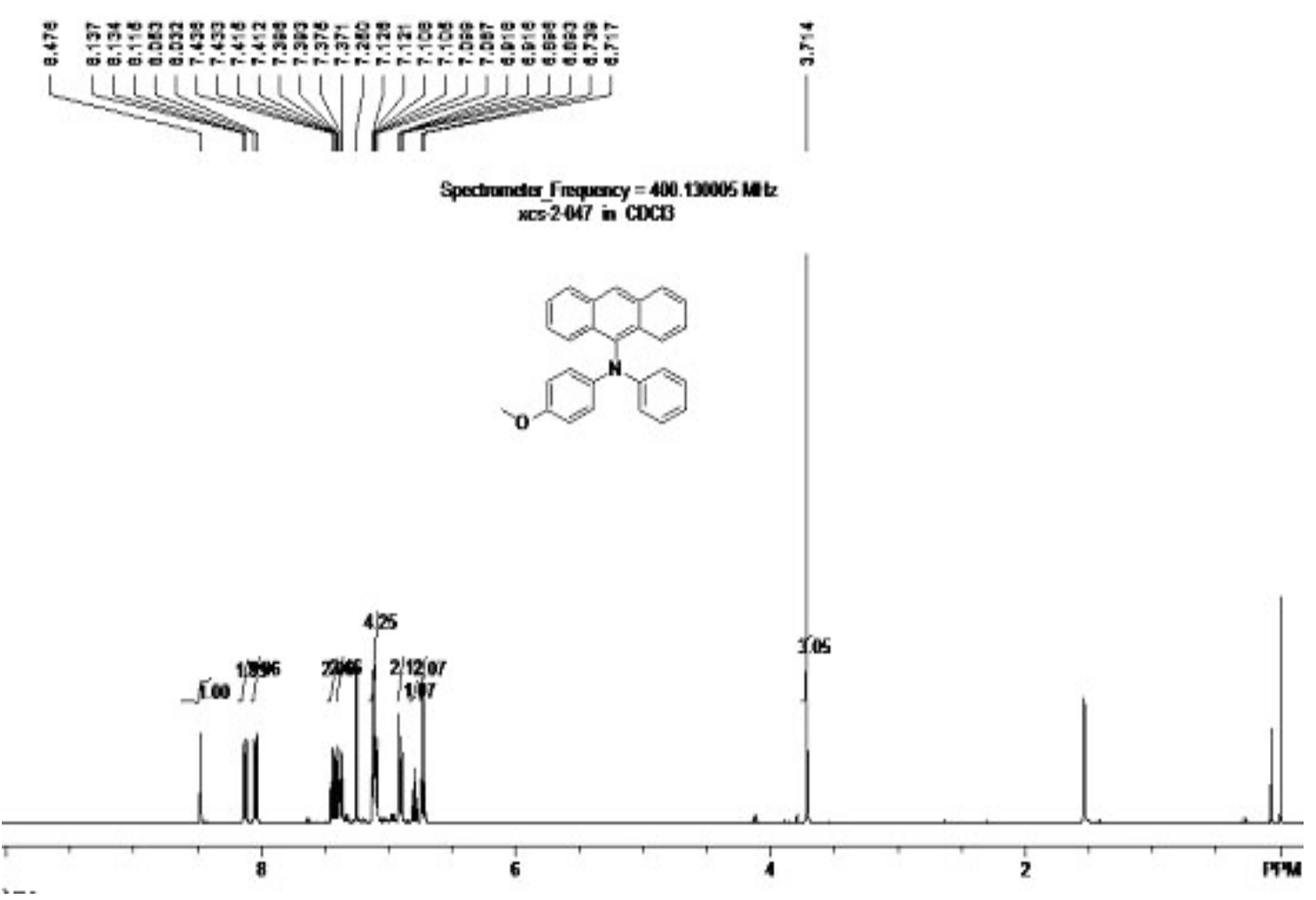




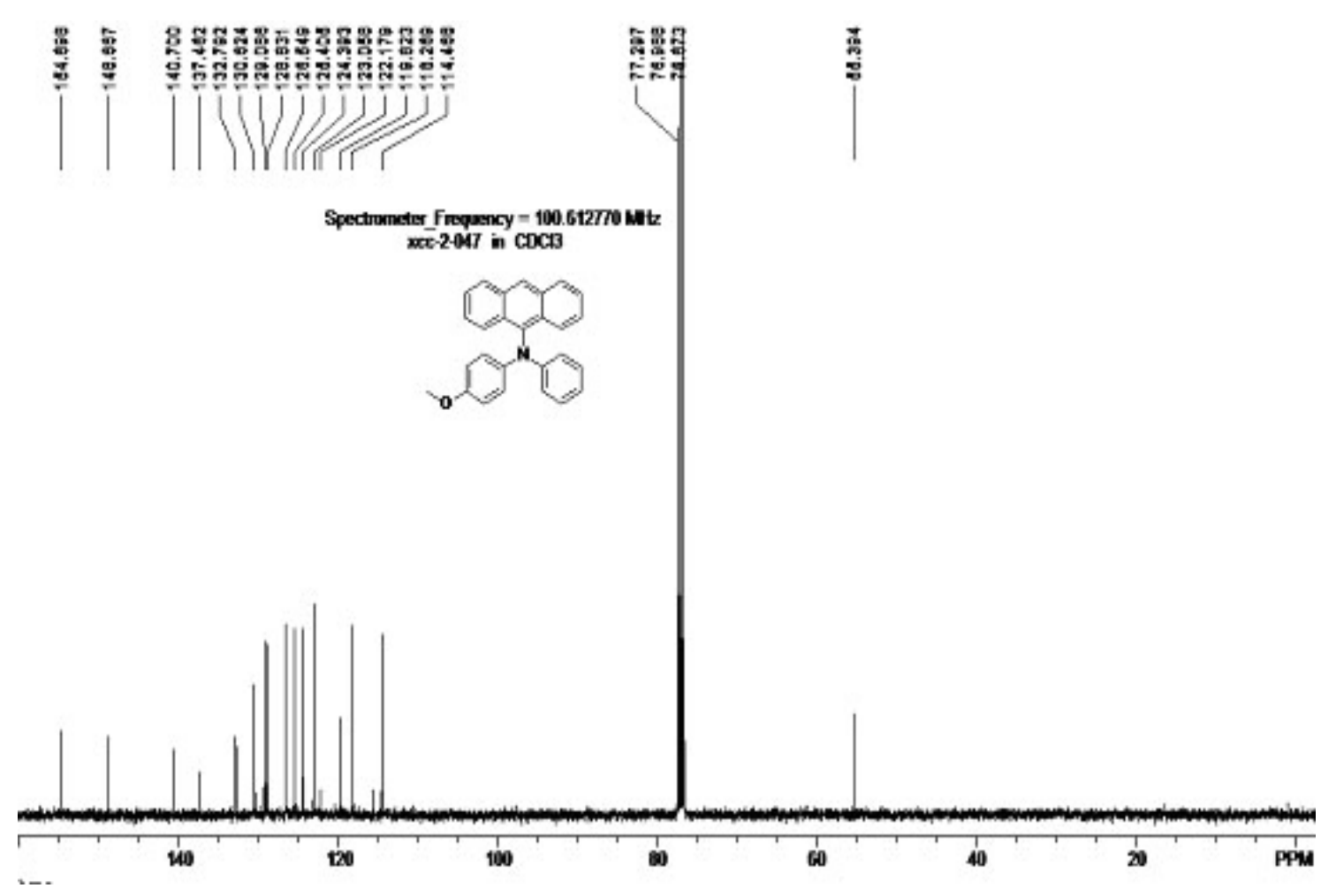

(8) N-phenyl-N-p-tolylanthracen-10-amine [ T 2-7, New compound ]

${ }^{1} \mathrm{H}$ NMR ( $400 \mathrm{MHz}, \mathrm{CDCl}_{3}$, TMS ) $\delta 8.49(\mathrm{~s}, 1 \mathrm{H}), 8.12(\mathrm{~d}, J=8.4 \mathrm{~Hz}, 2 \mathrm{H}), 8.05(\mathrm{~d}, J=8.4$ $\mathrm{Hz}, 2 \mathrm{H}$ ), 7.44 ( t $, J=7.4 \mathrm{~Hz}, 2 \mathrm{H}$ ), 7.38 ( t $J=7.8 \mathrm{~Hz}, 2 \mathrm{H}$ ), 7.13 ( t, $J=8 \mathrm{~Hz}, 2 \mathrm{H}$ ), 7.00 ( m, $6 \mathrm{H}), 6.83(\mathrm{t}, J=7.4 \mathrm{~Hz}, 1 \mathrm{H}), 2.24(\mathrm{~s}, 3 \mathrm{H}) .{ }^{13} \mathrm{C} \mathrm{NMR}\left(100 \mathrm{MHz}, \mathrm{CDCl}_{3}\right) \delta 148.1,145.0$, 137.3, 132.7, 130.8, 130.6, 129.7, 129.0, 128.8, 126.6, 126.5, 125.4, 124.3, 120.7, 120.4, 119.4, 20.4. IR ( KBr ) v = 3048, 3026, 2923, 1594, 1506, 1492, 1356, 1317, 1290, 879, 841, 812, 791, 747, 737, 691. HRMS (APCI) Calcd for $\mathrm{C}_{27} \mathrm{H}_{21} \mathrm{~N}$ : [M+H] ${ }^{+}$360.1747; Found, 360.1729. 


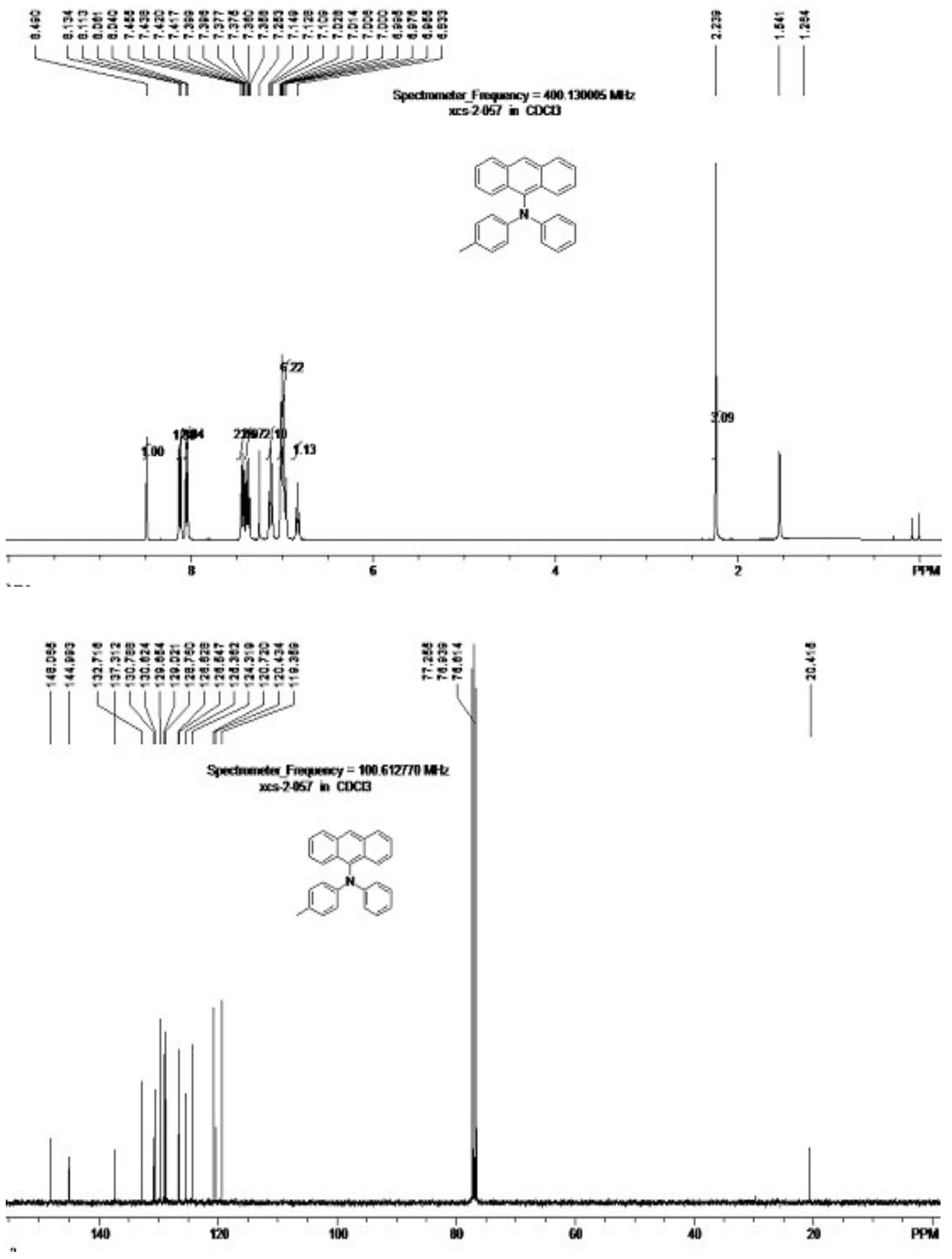




\section{(9) $\mathbf{N}$-(4-chlorophenyl)-N-phenylanthracen-10-amine [ T 2-8, New compound ]}

${ }^{1} \mathrm{H}$ NMR ( $400 \mathrm{MHz}, \mathrm{CDCl}_{3}$, TMS ) $\delta 8.51(\mathrm{~s}, 1 \mathrm{H}$ ), 8.07 ( s, $2 \mathrm{H}$ ), 8.05 ( s, $2 \mathrm{H}$ ), 7.45 ( t, J = $7.2 \mathrm{~Hz}, 2 \mathrm{H}$ ), 7.39 ( t $J=7.6 \mathrm{~Hz}, 2 \mathrm{H}$ ), 7.15 ( t $J=8 \mathrm{~Hz}, 2 \mathrm{H}$ ), 7.07 ( m, $4 \mathrm{H}$ ), 6.96 ( d, $J=8.8$ $\mathrm{Hz}, 2 \mathrm{H}$ ), 6.89 ( t, $J=7.2 \mathrm{~Hz}, 1 \mathrm{H}) .{ }^{13} \mathrm{C} \mathrm{NMR}\left(100 \mathrm{MHz}, \mathrm{CDCl}_{3}\right) \delta 147.2,146.3,136.6,132.7$, 130.4, 129.2, 129.1, 128.9, 127.1, 126.8, 125.6, 125.5, 123.9, 121.6, 121.0, 120.4. IR ( KBr ) v $=3052,1586,1488,1356,1307,1289,891,820,789,737,692$. HRMS (APCI) Calcd for $\mathrm{C}_{26} \mathrm{H}_{18} \mathrm{ClN}:[\mathrm{M}+\mathrm{H}]^{+}$380.1201; Found, 380.1188.
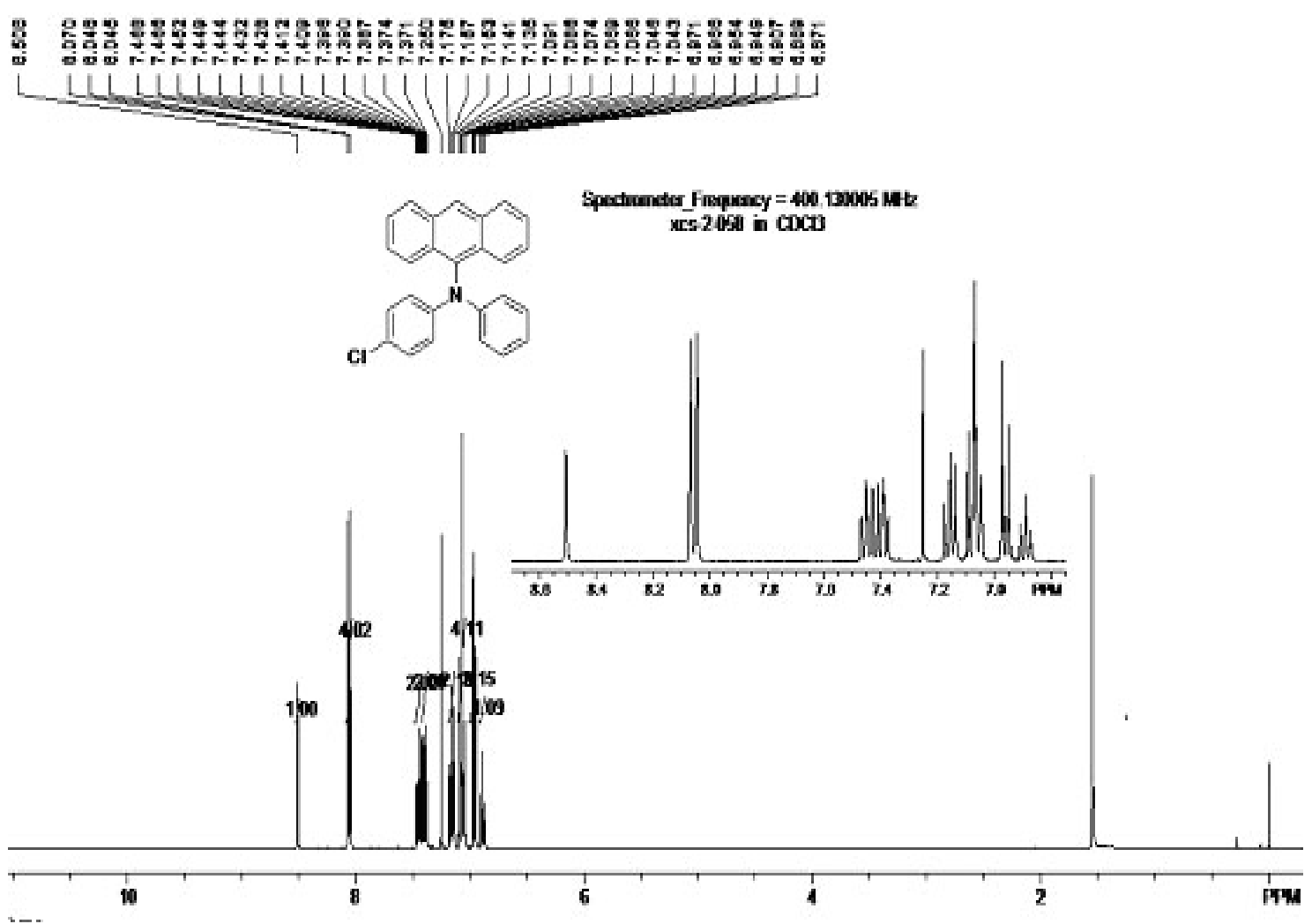


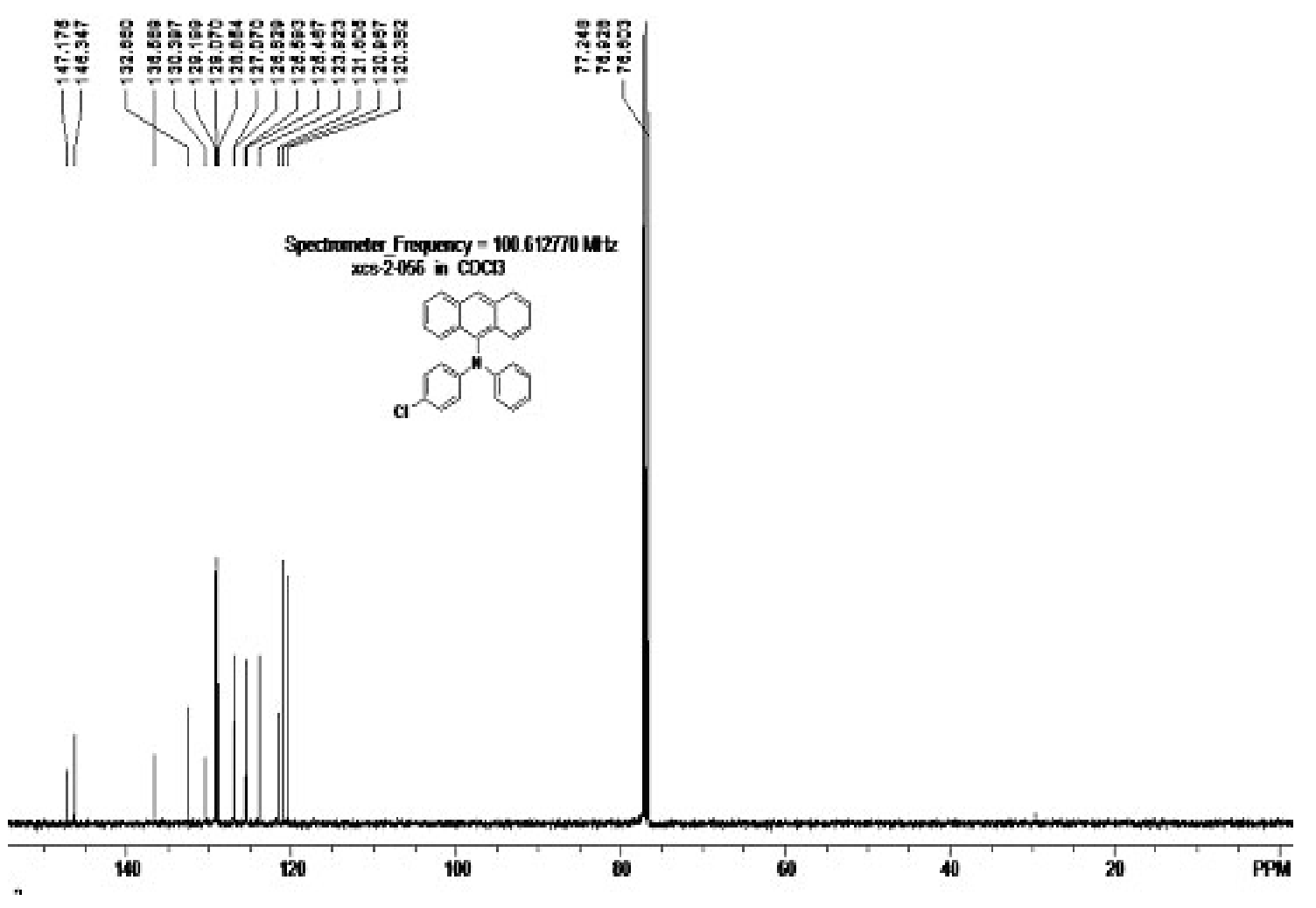

(10) N-phenyl-N-(4-(trifluoromethyl)phenyl)anthracen-10-amine [ T 2-9, New compound ] ${ }^{1} \mathrm{H}$ NMR ( $400 \mathrm{MHz}, \mathrm{CDCl}_{3}$, TMS ) $\delta 8.54$ ( s, $1 \mathrm{H}$ ), 8.06 ( t, $J=9.4 \mathrm{~Hz}, 4 \mathrm{H}$ ), 7.47 ( t, $J=7.4$ $\mathrm{Hz}, 2 \mathrm{H}$ ), 7.41 ( t, $J=7.6 \mathrm{~Hz}, 2 \mathrm{H}$ ), 7.35 ( d, J = 8.8 Hz, $2 \mathrm{H}$ ), 7.20 ( m, 4 H ), 6.98 ( m, $3 \mathrm{H}$ ). ${ }^{13} \mathrm{C}$ NMR ( $100 \mathrm{MHz}, \mathrm{CDCl}_{3}$ ) $\delta 150.8,146.3,136.2,132.7,130.3$ ( q, $J=29.5 \mathrm{~Hz}$ ), 129.4, 129.0, 127.5, 127.2, 126.5 ( q, $J=3.7 \mathrm{~Hz}$ ), 125.6, 124.6 ( q, $J=268.5 \mathrm{~Hz}$ ), 123.8, 123.0, 122.0, 121.6, 118.0. IR ( KBr ) v = 3055, 2924, 2847, 1612, 1592, 1515, 1496, 1321, 1298, 1112, 1066, 895, 828, 736, 695. HRMS (APCI) Calcd for $\mathrm{C}_{27} \mathrm{H}_{18} \mathrm{~F}_{3} \mathrm{~N}$ : [M+H $]^{+}$414.1464; Found, 414.1441. 


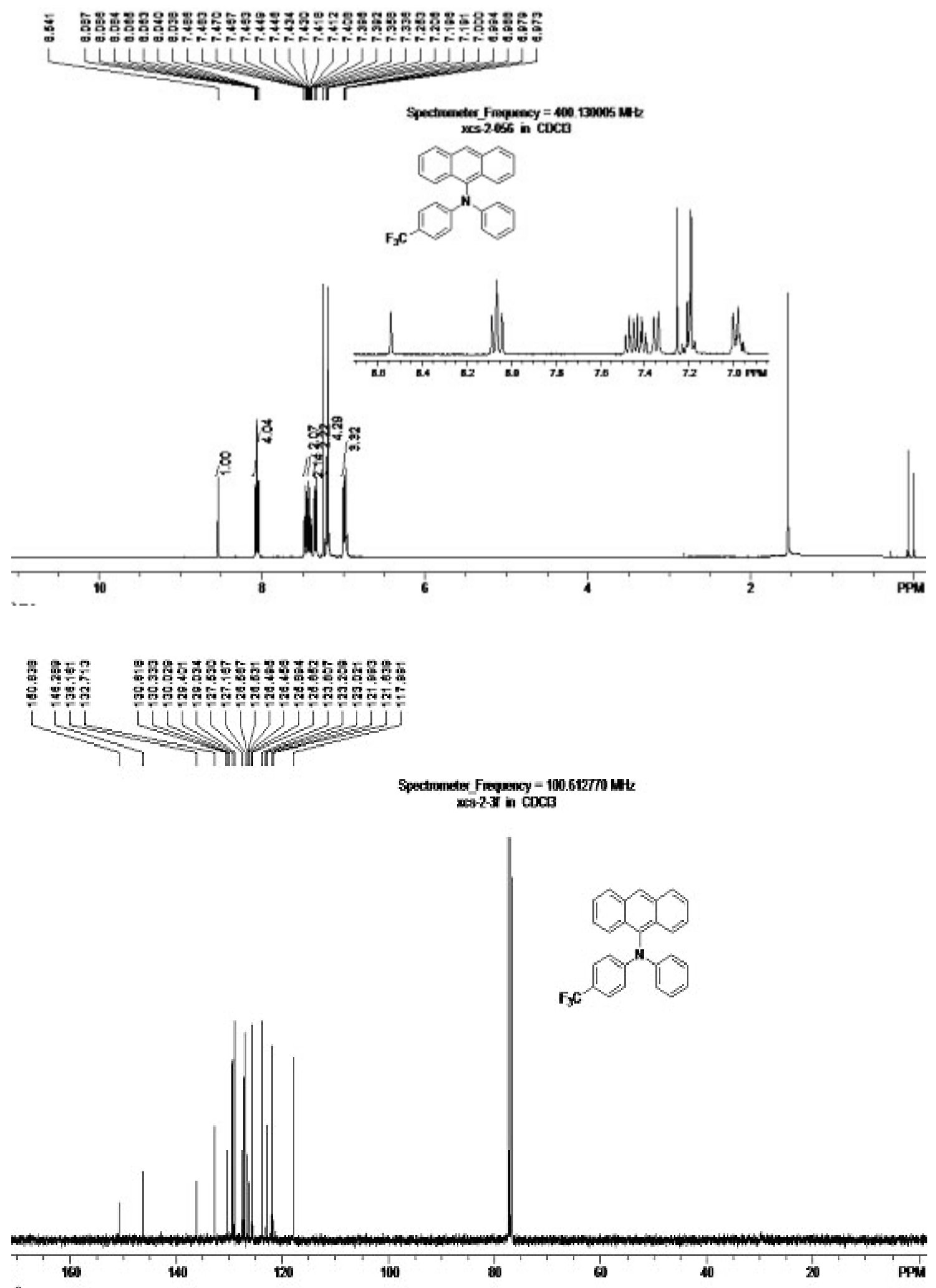


(11) N,9-dimethyl-N-phenylanthracen-10-amine [ T 2-10, New compound ]

${ }^{1} \mathrm{H}$ NMR ( $400 \mathrm{MHz}, \mathrm{CDCl}_{3}$, TMS ) $\delta 8.38(\mathrm{~d}, J=8.8 \mathrm{~Hz}, 2 \mathrm{H}$ ), $7.95(\mathrm{~d}, J=8 \mathrm{~Hz}, 2 \mathrm{H}$ ), 7.53 ( t $J=7.4 \mathrm{~Hz}, 2 \mathrm{H}$ ), 7.43 ( t $, J=7.6 \mathrm{~Hz}, 2 \mathrm{H}$ ), 7.14 ( s, $2 \mathrm{H}$ ), 6.69 ( t, $J=7.4 \mathrm{~Hz}, 1 \mathrm{H}$ ), 6.49 ( $\mathrm{s}$, $1 \mathrm{H}) .{ }^{13} \mathrm{C} \mathrm{NMR}\left(100 \mathrm{MHz}, \mathrm{CDCl}_{3}\right.$ ) $\delta 150.0,137.9,131.1,130.2,129.5,129.1,128.1,127.1$, 125.9, 125.4, 124.7, 116.3, 39.4, 14.2. IR ( KBr ) v = 3056, 2924, 2847, 1649, 1595, 1500, 1392, 1326, 1286, 759, 743, 692. HRMS (APCI) Calcd for $\mathrm{C}_{22} \mathrm{H}_{19} \mathrm{~N}$ : $[\mathrm{M}+\mathrm{H}]^{+}$298.1590; Found, 298.1576.

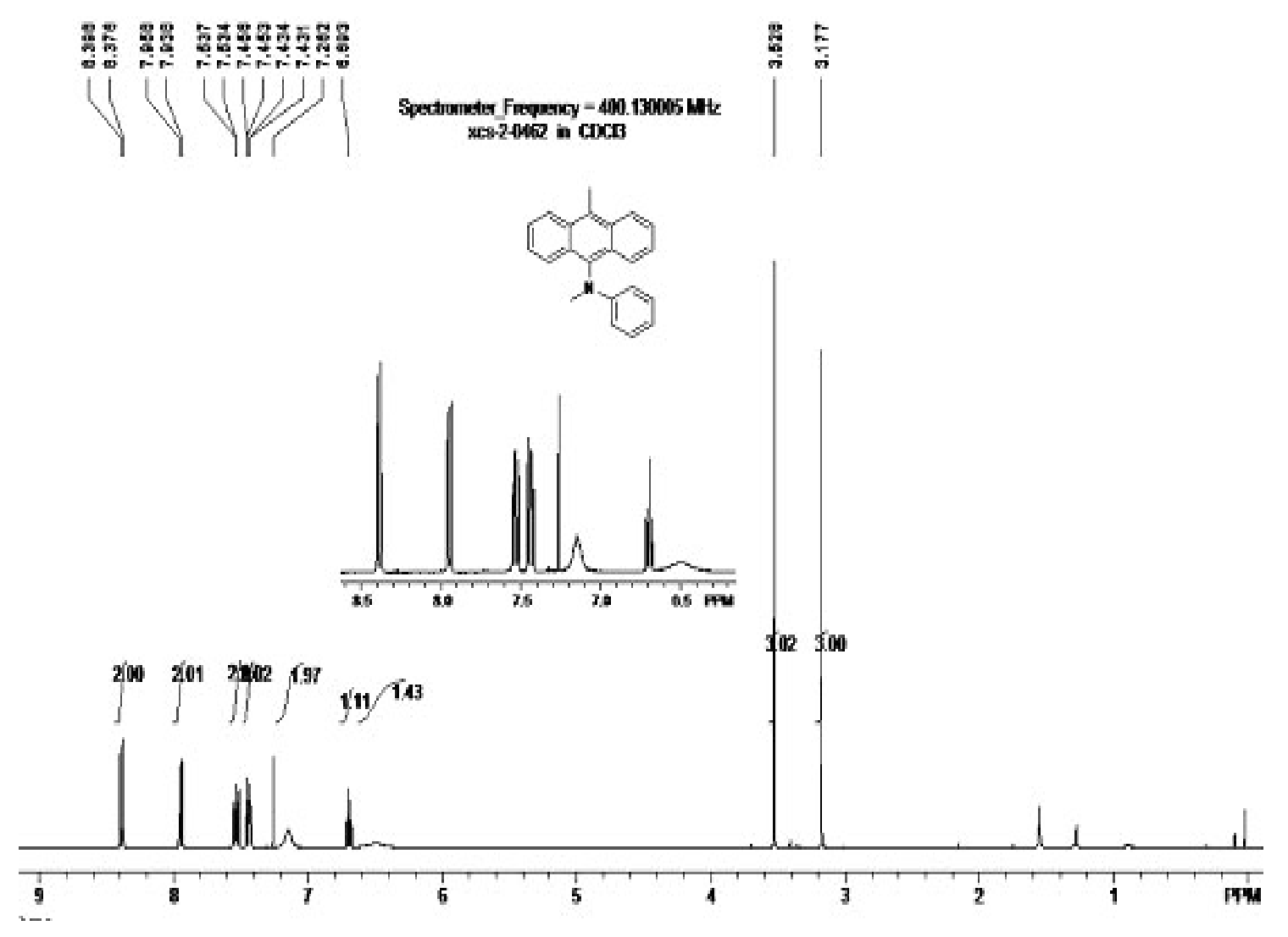




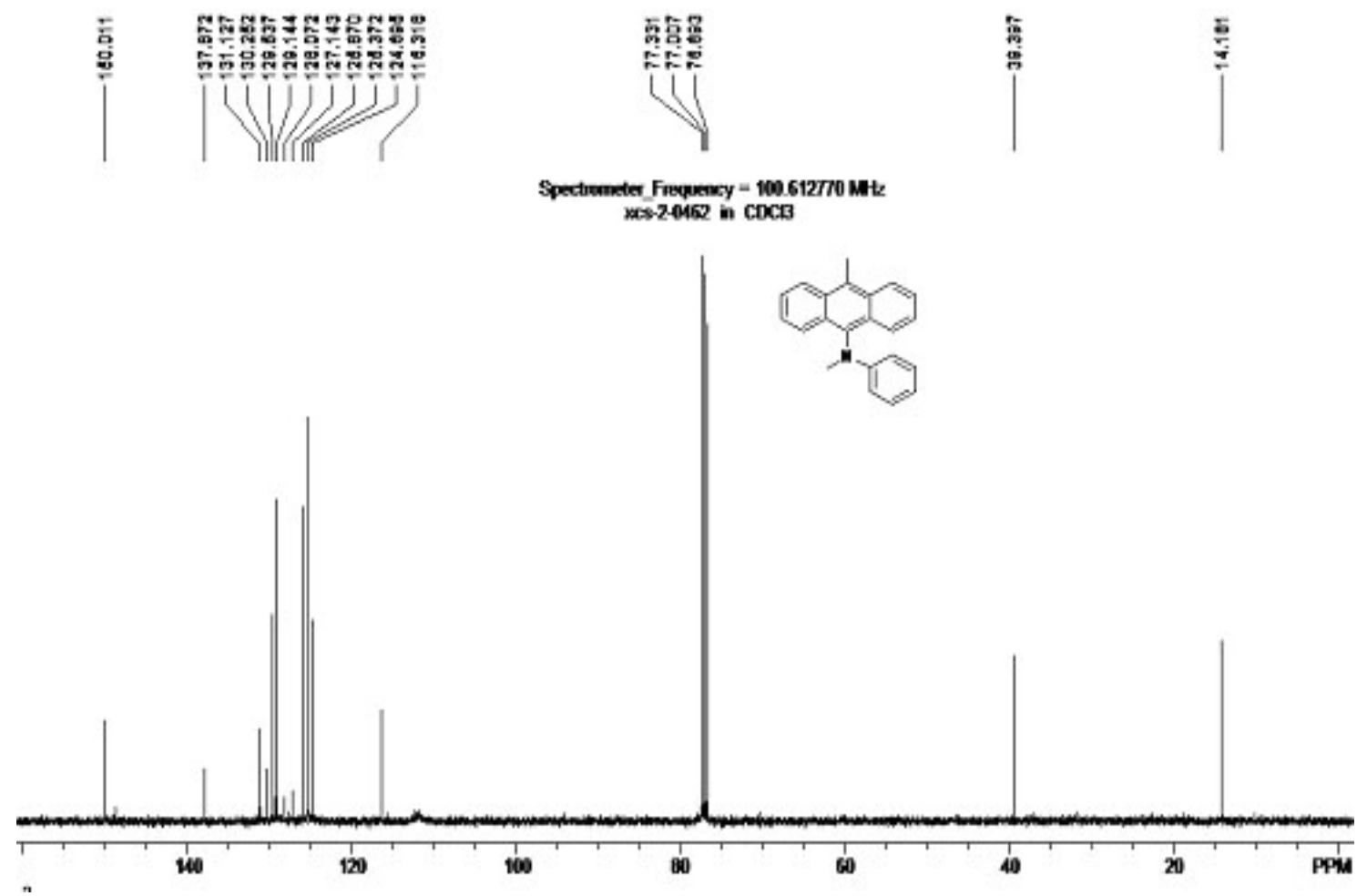

(12) N-ethyl-N,9-diphenylanthracen-10-amine [ T 2-11, New compound ]

${ }^{1} \mathrm{H} \mathrm{NMR}\left(400 \mathrm{MHz}, \mathrm{CDCl}_{3}\right.$, TMS ) $\delta 8.02(\mathrm{~d}, J=8.8 \mathrm{~Hz}, 2 \mathrm{H}$ ), $7.72(\mathrm{~d}, J=8.8 \mathrm{~Hz}, 2 \mathrm{H}$ ), 7.59 $(\mathrm{m}, 3 \mathrm{H}), 7.51(\mathrm{~d}, J=8 \mathrm{~Hz}, 1 \mathrm{H}), 7.47(\mathrm{~d}, J=6.8 \mathrm{~Hz}, 1 \mathrm{H}), 7.40(\mathrm{t}, J=7.8 \mathrm{~Hz}, 2 \mathrm{H}), 7.34(\mathrm{t}$, $J=7.6 \mathrm{~Hz}, 2 \mathrm{H}$ ), $7.16\left(\mathrm{t}, J=8.2 \mathrm{~Hz}, 2 \mathrm{H}\right.$ ), 6.70 ( t $J=7.4 \mathrm{~Hz}, 1 \mathrm{H}$ ), 6.56 ( s, $2 \mathrm{H}$ ). ${ }^{13} \mathrm{C} \mathrm{NMR}$ $\left(100 \mathrm{MHz}, \mathrm{CDCl}_{3}\right) \delta 149.3,138.7,138.1,137.0,131.4,131.3,131.2,130.1,129.2,128.4$, 127.6, 125.9, 125.3, 124.5, 116.3, 112.0, 47.4, 13.6. IR ( KBr ) $v=3064,2962,2926,1598$, 1500, 1398, 1274, 774, 746, 703, 608. HRMS (APCI) Calcd for $\mathrm{C}_{28} \mathrm{H}_{23} \mathrm{~N}$ : $[\mathrm{M}+\mathrm{H}]^{+} 374.1903$; Found, 374.1883. 


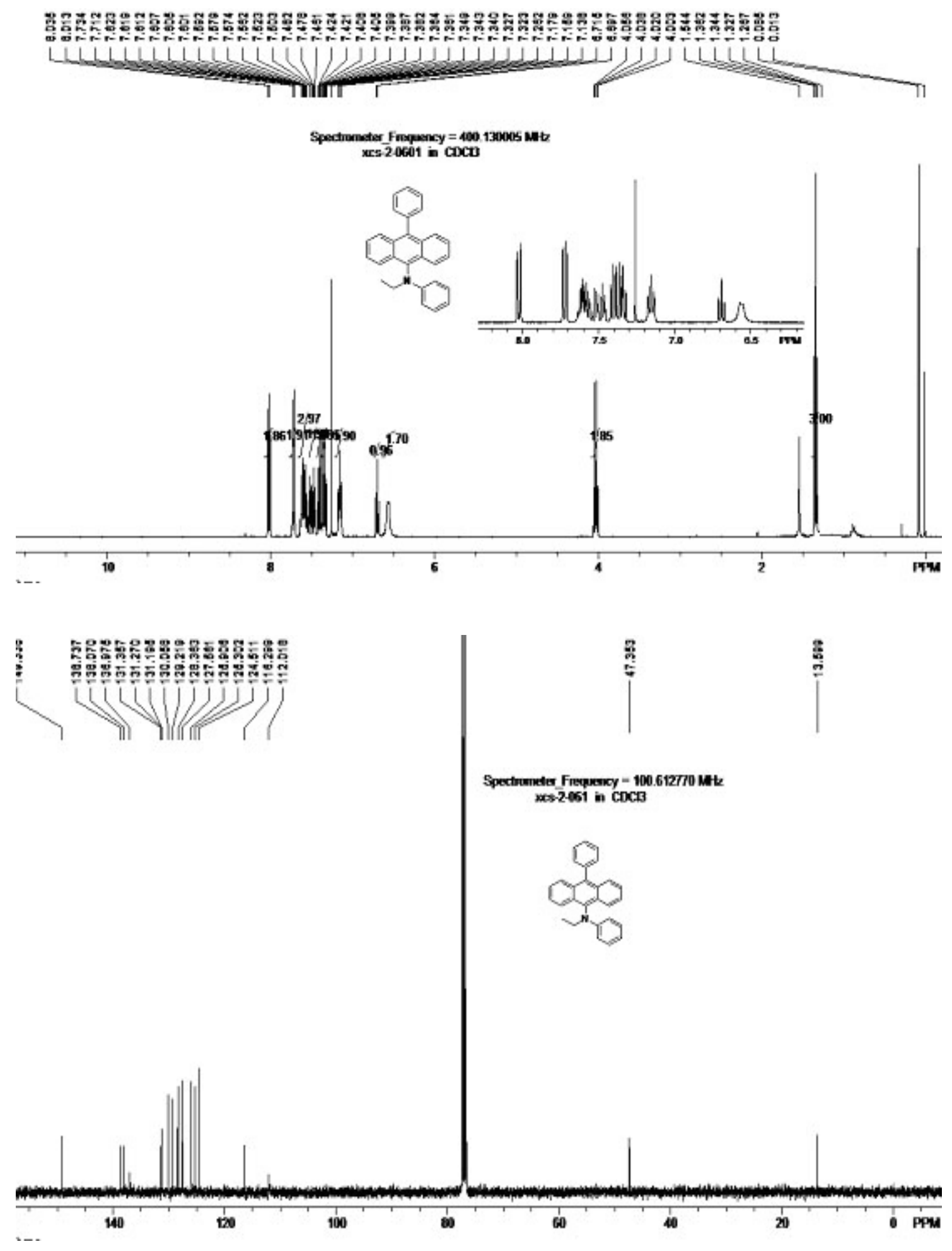

GC-MS analysis of the reaction solution of o-trimethylsilyl aryltriflate 
with 1-ethyl-4-phenyl-1H-imidazole (T2-12)

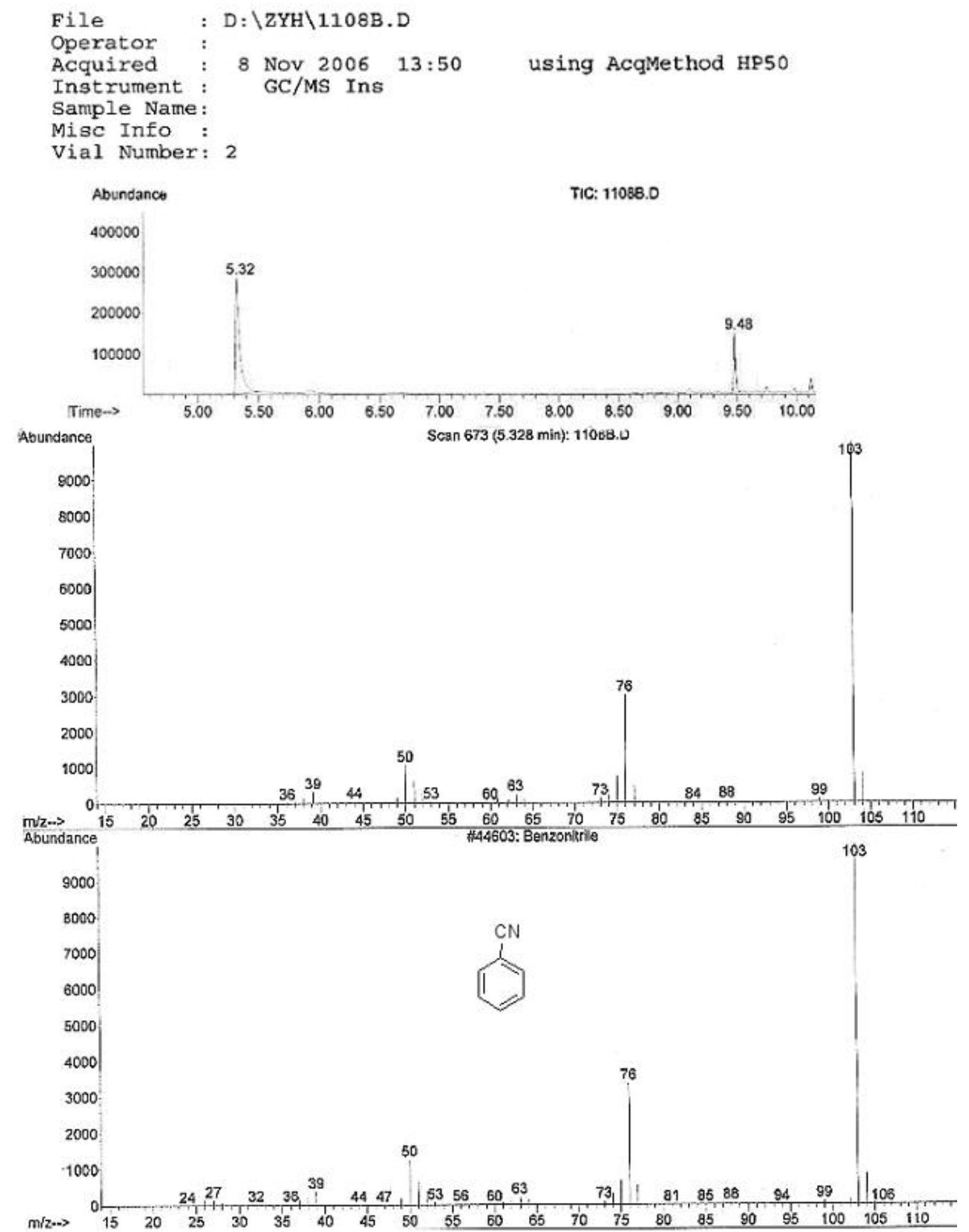




\section{Refereneces}

(1) Himeshima, Y.; Sonoda, T.; Kobayashi, H. Chem. Lett. 1983, 1211-1214.

(2) Bonhôte, P.; Dias, A. P.; Papageorgiou, N.; Kalyanasundaram, K.; Grätzel, M. Inorg. Chem. 1996, $35,1168-1178$.

(3) Klapars, A.; Antilla, J. C.; Huang, X. H.; Buchwald, S. L. J. Am. Chem. Soc. 2001, 123, $7727-7729$.

(4) Tamano, M.; Okutsu, S.; Enokida, T. Jpn. Kokai Tokkyo Koho 1997, 11. 